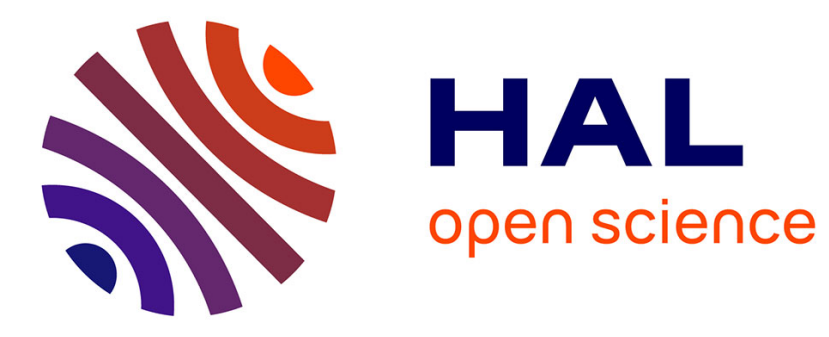

\title{
Investigating the Impact of Partial Topology in Proactive MANET Routing Protocols
}

Thomas Clausen, Philippe Jacquet, Laurent Viennot

\section{To cite this version:}

Thomas Clausen, Philippe Jacquet, Laurent Viennot. Investigating the Impact of Partial Topology in Proactive MANET Routing Protocols. The 5th International Symposium on Wireless Personal Multimedia Communications (WPMC), Oct 2002, Honolulu, United States. 10.1109/WPMC.2002.1088405 . inria-00471706

\section{HAL Id: inria-00471706 https://hal.inria.fr/inria-00471706}

Submitted on 8 Apr 2010

HAL is a multi-disciplinary open access archive for the deposit and dissemination of scientific research documents, whether they are published or not. The documents may come from teaching and research institutions in France or abroad, or from public or private research centers.
L'archive ouverte pluridisciplinaire HAL, est destinée au dépôt et à la diffusion de documents scientifiques de niveau recherche, publiés ou non, émanant des établissements d'enseignement et de recherche français ou étrangers, des laboratoires publics ou privés. 


\title{
Investigating the Impact of Partial Topology in Proactive MANET Routing Protocols
}

\author{
Thomas Heide Clausen. ${ }^{*}$, Philippe Jacquet, Laurent viennot \\ Project HiPERCOM, INRIA Rocquencourt \\ B.P. 105, 78153 Le Chesnay Cedex, France \\ \{thomas.clausen, Philippe Jacquet, laurent.viennot\}@inria.fr
}

\section{Keywords}

Wireless Ad-Hoc Networks, Partial Topology, OLSR, Performance Evaluation, Simulation.

\begin{abstract}
In this paper, we study the impact of using partial versus full topology in the OLSR routing protocol for MANETs. The core of OLSR is the notion of MultiPoint Relays (MPRs), serving the purpose of reducing the amount of link-state information, flooded to nodes in the network, as well as reducing the redundancy in the flooding process.

The OLSR protocol specification contains a tunable parameter, MPR coverage, which adjusts the degree of redundancy in both the advertised link-state information, and the links over which the link-state information is advertised. We investigate the impact from assigning various values to this parameter. We further investigate two options for advertising additional link-state information: the "MPR full link-state" option implies that whenever a node is selected to declare any link-state information, it declares all its local link state information. The "full link-state" option states, that all nodes must declare all their local link-state information.

Through simulations, we investigate the performance characteristics of OLSR with and without these options.
\end{abstract}

\section{INTRODUCTION}

A mobile ad-hoc network (MANET) is a collection of nodes, connected by wireless links, forming an arbitrary, dynamic graph. The wireless medium typically implies a bandwidthconstrained network. This due to the lower bandwidth provided by the network adaptors and to the fact that communication over any link will interfere with communication on any other link within radio range.

Mobility implies that links between nodes may change and that the number of nodes in a network is not constant. The physical size of a MANET is expected to be larger than the radio range of the wireless interfaces, thus for any two nodes in the network to be able to communicate, routing is necessary.

\footnotetext{
${ }^{*}$ Thomas Clausen may also be contacted at MindPass Center for Distributed Systems, Department of Computer Science, Aalborg University,Frederik Bajers Vej 7E, 9220 Aalborg, Denmark
}

\section{Mobile Ad Hoc Network routing protocols}

Two requirements are presented for MANET routing protocols: the ability to maintain routes, despite a dynamic topology, while economizing bandwidth consumption.

Two classes of MANET routing protocols exist: reactive protocols, including DSR [2] and AODV [5], discovers and maintains routes only when required through a request-reply flooding cycle. Proactive protocols, including OLSR [6] [8] [7], FSR [3] and TBRPF [4], maintain routes to all destinations at all times through periodic advertisements. In this paper, we will be concerned only with proactive protocols.

A common characteristic among these proactive protocols is, that they seek to reduce overhead by maintaining partial topological information. While all destinations in a network are known by all nodes, OLSR and TBRPF maintains partial link state information in all nodes, and diffuse such information using only a subset of the links in the network. FSR introduces temporal partial information: information about a link is maintained in all nodes; the frequency at which this information is updated in a node depends on the nodes distance to the link.

In this paper, we investigate the impact of using partial topological information in a MANET routing protocol. We base our investigations on OLSR.

\section{The Optimized Link State Routing Protocol}

OLSR [6] employs periodic flooding of topological information, contained in TC-messages, to all nodes in the network. Flooding is performed using the concept of multipoint relays (MPRs) [1]. Each node selects among its neighbors a set of nodes, called "multipoint-relays". This set is selected such that any node in the 2-hop neighborhood is reachable through at least one MPR. A node periodically declares its MPRs to its neighborhood, whereby each node learns about its "MPR selectors" - the set of neighbor nodes which have selected a given node as MPR..

When a TC-message is received by a node, the information contained herein is recored and used for building the routing table in the node. The TC-message is then forwarded under two conditions: the message has not been seen before by the node and the node is selected as MPR for the "last hop" of the message. By using this mechanism, denoted "MPR Flooding", all nodes in the network will (disregarding loss due to collisions etc.) receive a given TC-message. With a minimal MPR-set, the number of transmissions of a given message in an area is significantly reduced as compared to 
classical flooding.

Another optimization is, that TC-messages are generated only by nodes with a non-empty set of MPR selectors. A TC-messages contains a list of links between the node and its MPR selectors. Since all reachable nodes will select a non-empty set of MPRs, all reachable destinations will thus be declared in the network - yet only a partial set of the links in the network will be declared. This reduces the size of the control packets being flooded and, since only a subset of all nodes are selected as MPR, reduces the number of nodes flooding link-state information as well.

Thus OLSR can be said to be a partial link-state protocol in three ways: only a subset of all links in the network are declared in TC-messages, only a subset of nodes generate TC-messages, and TC-messages are flooded to all nodes, utilizing only a subset of the links available.

\section{Paper outline}

In this paper, we will investigate the impacts of using partial topology in OLSR. Specifically, we will be investigating two issues: the impact of selecting a minimal MPR versus redundant MPR set, as well as the impact of advertising partial versus full link-state information.

Thus, the remainder of this paper will be as follows: in the next section, we describe different partial and full topology options for OLSR. Following, we present simulations, illustrating how these options affect the performance and the overhead of the protocol. Our simulations include both simulations with an idealized model for the physical layer and for mobility, as well as ns2-simulations. Finally, the paper is concluded.

\section{TUNABLE PARTIAL TOPOLOGY IN OLSR}

The Optimized Link State Routing protocol, as described in the introduction, aims at reducing the control traffic overhead through being a partial link-state protocol. This is achieved through each node minimizing its MPR set, thereby both minimizing the amount of link-state information advertised and minimizing the set of links over which this information is transmitted.

In this section, we describe ways in which the OLSR protocol can be tuned, allowing for different degrees of partial and full topology.

\section{MPR coverage}

In OLSR, the "MPR coverage" is a tunable parameter, specifying the degree of redundancy in the MPR selection. With an MPR coverage of 1, a minimal MPR-set is sought, minimizing both overhead and redundancy. We will denote OLSR with MPR coverage of 1 "regular OLSR".

With an MPR coverage of $\mathrm{k}$, it is sought that any node in the 2-hop neighborhood is reachable through at least $\mathrm{k}$ MPRs - implying increased overhead and redundancy: for MPR coverage $>1$, redundant link-state information is included in the TC-messages, more nodes will emit TC-messages and TC-messages are flooded through a redundant set of links in the network. I.e. a node may receive copies of the same TC-message over multiple links.

\section{MPR full link-state}

We specify an "MPR full link-state" option with the purpose of increasing the amount of redundant topological information included in TC-messages, without increasing the number of TC-messages or the number of links over which the message is flooded:

each node, selected by any node as MPR, emits

TC-messages, declaring all links between the node itself and all neighbor nodes.

This will not cause more TC-messages to be emitted, when compared to regular OLSR, however will imply an increased size of each TC-message. Since the MPR selection is not affected, the TC messages will be flooded using the optimized MPR flooding.

\section{Full link-state}

To allow that all links in the network be declared, we specify

"full link-state" option as follows: each node emits TC-messages, declaring all links between the node itself and all neighbor nodes.

This will provide all nodes with full topological information, on expense of an increase in both the number and size of TCmessages. Since the MPR selection is not affected, the TC messages will be flooded using the optimized MPR flooding.

\section{SIMULATIONS AND RESULTS}

To evaluate and compare the impact of the various options for partial and full topology in OLSR, exhaustive simulations are conducted. In this section, we describe the simulation parameters, and present the results of our simulations.

\section{Simulation parameters}

For all situations, we conduct exhaustive ns2-simulations. For each sample point, 30 random scenarios are generated, corresponding to the parameters describing that point. The simulation results presented are an average over these 30 scenarios. This reduces the chance that results are dominated by a single scenario which, accidentally, favors one protocol over another. We emphasize, that the same set of 30 scenarios are used for all simulations in a given sample point, hence the different protocol options are evaluated under identical conditions.

The parameters used in the ns 2 simulations are included in table 1.

For the MPR coverage evaluation, we also conduct custom simulations with direct control over the error probability of a broadcast transmission over a link. The model used in these simulations assumes a fixed probability of error when receiving a broadcast packet. The fraction of nodes actually receiving a message broadcasted by flooding depend on this probability. This measure is used to estimate the reliability of flooding schemes. For the simulations conducted using this model, we assume the same basic parameters as for the 


\begin{tabular}{|ll|}
\hline \multicolumn{2}{|c|}{ Simulator } \\
\hline Propagation model & TwoRayGrers \\
Network type & IEEE $802.11(2 \mathrm{Mbps})$ \\
Transmission range & $250 \mathrm{~m}$ \\
\hline \multicolumn{2}{|c|}{ Scenario parameters } \\
\hline Field size & $1500 \mathrm{~m} \times 300 \mathrm{~m}^{2}$ \\
Number of nodes & 50 \\
Simulation time & 300 seconds \\
Mobility model & Random waypoint \\
Node speed & Variable, see graphs \\
Node rest time & 5 seconds \\
Movement distance & $30 \mathrm{~m}$ \\
\hline \multicolumn{2}{|c|}{ Test traffic parameters } \\
\hline Number of streams & 20 seconds \\
Traffic rate pr stream & 640 bytes $/ \mathrm{seconds}$ \\
Stream duration & 30 seconds \\
\hline
\end{tabular}

Table 1. Simulator and Scenario parameters.

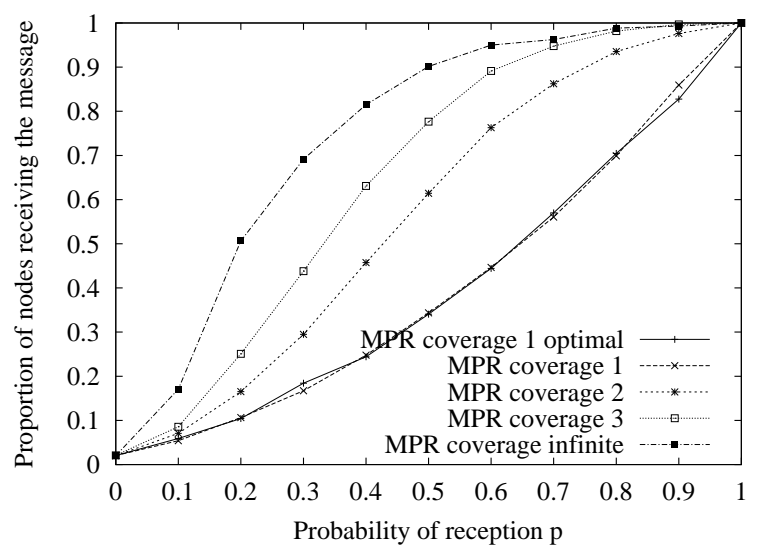

Figure 1. Delivery rate versus link reliability.

the ns 2 simulations, i.e. a field size of $1500 \times 300 \mathrm{~m}^{2}$ with 50 nodes.

\section{MPR coverage simulations}

Figure 1 shows the fraction of nodes which receives a packet, flooded using MPR flooding, under variable probability of receiving a broadcast message over a link. We observe, that the reliability of an MPR flooding can be smoothly increased by increasing MPR coverage parameter.

In OLSR, the packets being subject to MPR flooding contain TC-messages. Figure 2 shows the delivery rate of TCmessages, under variable mobility. The figure shows delivery rates for MPR coverage ranging from 1 to 7 . We observe, that by increasing the MPR coverage, we can graduately increase the delivery rate of TC-messages, confirming figure 1.

Figure 3 shows the total control traffic overhead, incurring from TC-messages. We firstly note, that the version of OLSR used for these simulations does not react explicitly to link breakage and thus that the control traffic is constant - inde-

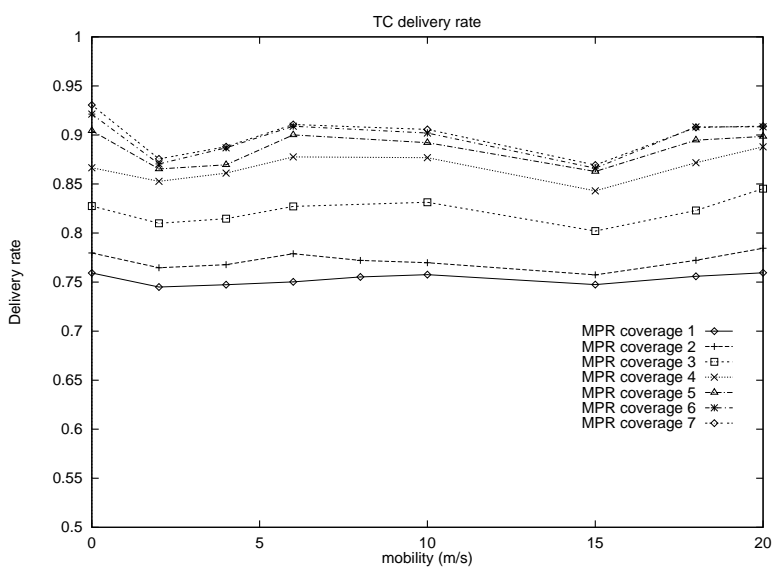

Figure 2. Delivery rate of TC-messages from ns2 simulations.

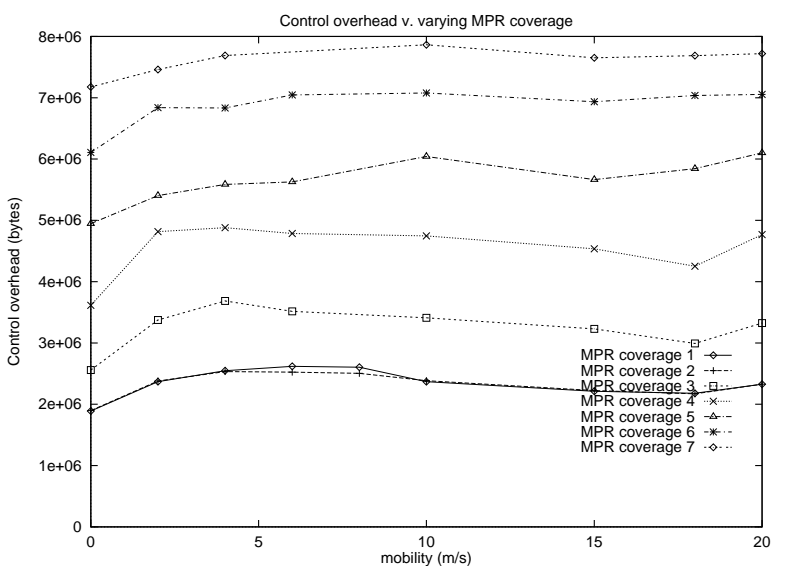

Figure 3. Control traffic overhead from ns2 simulations.

pendent of mobility.

Secondly, we observe that the control traffic overhead is increasing graduately with the the MPR coverage. The control traffic overhead is practically identical for MPR coverage 1 and 2. This means that even with MPR coverage $=1$, most two-hop nodes in this scenario are covered by 2 MPRs.

This is confirmed by the TC delivery rate, illustrated in figure 2, where the TC-message delivery rate for MPR coverage of 1 and 2 are almost identical.

Figure 4 shows the delivery rate for data traffic with different MPR coverage. We observe, that except for in high-mobility scenarios, there is no significant difference in the data delivery rates. This indicates that, for the scenario tested, an MPR coverage of 1 , regular OLSR, is sufficient to provide nodes with the required topological information.

\section{MPR full link-state and full link-state}

Figure 5 shows the control traffic overhead for regular OLSR (MPR coverage $=1$, MPR nodes advertise links to their MPR selector set in TC-messages), as well as for the MPR full link-state and full link-state options described previously. 


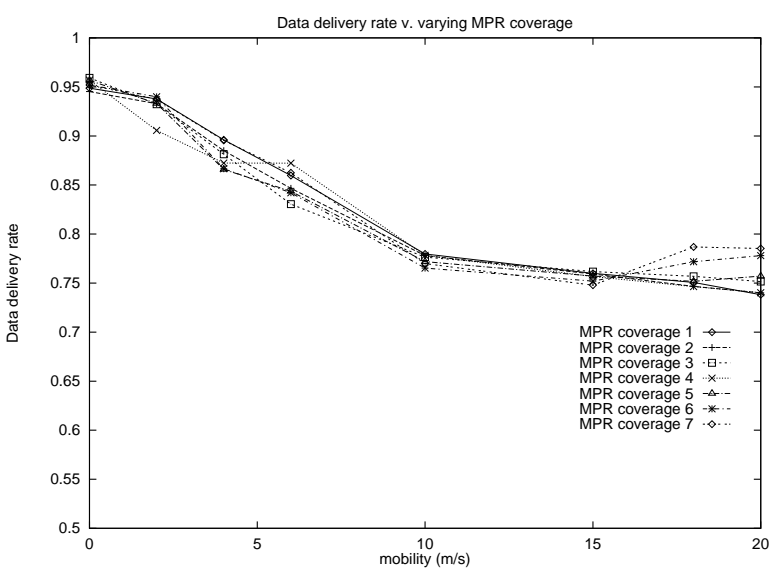

Figure 4. Data delivery rate from ns2 simulations.

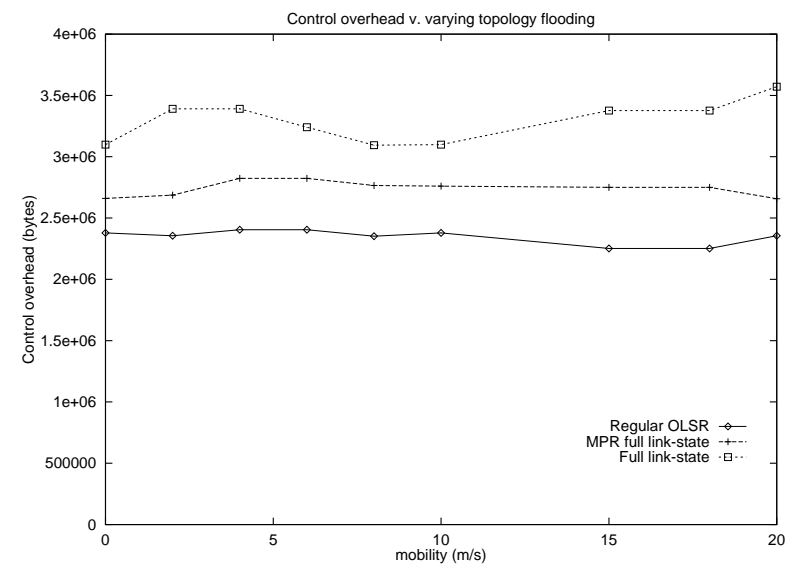

Figure 5. Control traffic overhead with varying topology from ns2 simulations.

As could be expected, the control traffic overhead from regular OLSR remains the lowest, while the control traffic overhead from the full link-state option yields the highest overhead.

Figure 6 shows the TC delivery rate for regular OLSR, as well as for OLSR with each of the two options. Since the same MPR flooding mechanism is employed, roughly identical delivery rates of TC-messages is observed.

Figure 7 shows the data delivery rates for regular OLSR, as well as for OLSR with each of MPR full link-state and full link-state enabled. We observe that the data delivery rates for the MPR full link-state and full link-state options are higher than for regular OLSR. We also observe, that the delivery rate of OLSR starts dropping at a lower mobility than the delivery rates of either of the two options. We note, that the additional topological information makes the routing more robust to mobility. The largest difference in delivery rate occurs between $8-10 \mathrm{~m} / \mathrm{s}$ where the MPR full link-state option yields a $13 \%$ higher delivery rate than regular OLSR and where the full link-state option yields a $20 \%$ higher delivery rate. This benefit comes at the expense of an increased

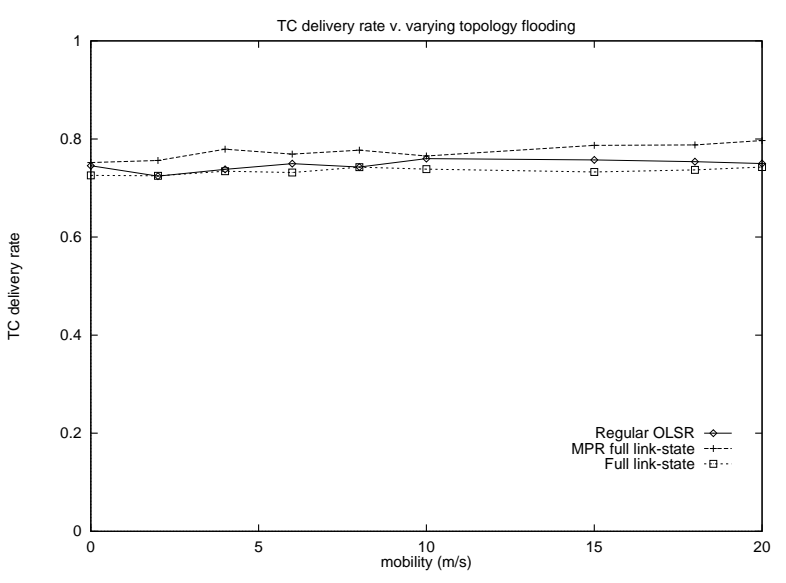

Figure 6. TC delivery rate with varying topology flooding mechanisms from ns2 simulations.

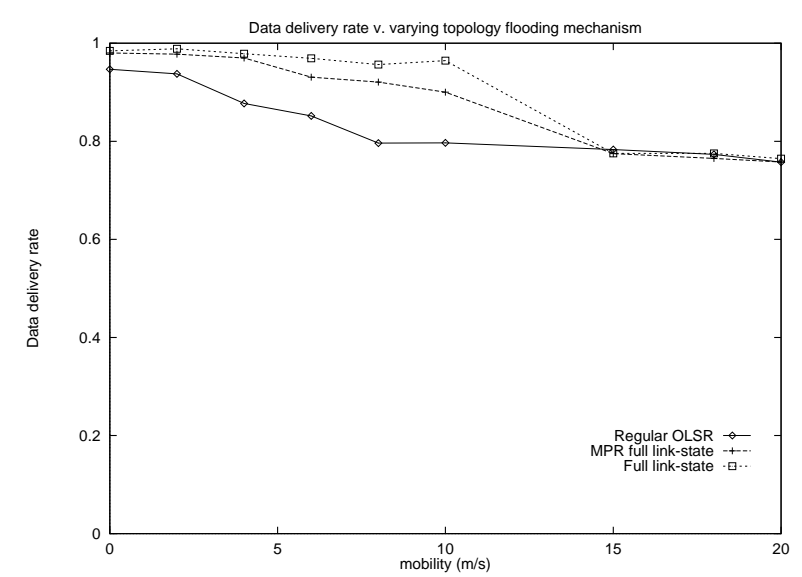

Figure 7. Data delivery rate with varying topology flooding mechanisms from ns2 simulations.

bandwidth consumption: with the MPR full link-state option, $16 \%$ more bandwidth is consumed by control traffic, while with the full link-state option, the bandwidth consumption is $30 \%$ higher than that of regular OLSR.

At about $15 \mathrm{~m} / \mathrm{s}$, the delivery rate of regular OLSR levels with that of both full topology options.

We finally notice, that even for the case of very high mobility $(20 \mathrm{~m} / \mathrm{s})$ delivery rates of above 0.75 are maintained.

\section{CONCLUSION}

Three different options for tuning the degree of partial topology utilized in OLSR are presented.

MPR coverage provides a way in which to tune the redundancy of MPR flooding for achieving better reliability at the cost of more emissions. We observe conclusively that, indeed, increasing the MPR coverage does increase the delivery rate of flooded TC-messages. In terms of data delivery, our simulations indicate, that the redundancy of topological information and retransmissions provided by a higher MPR coverage may provide better data delivery in high-mobility 
situations. However further studies are required to provide conclusive information confirming this.

We present two options, enabling additional link-state information to be diffused in the network. The MPR full link-state option specifies that nodes selected as MPR, when emitting a TC message, include links to all their neighbor nodes. The full link-state option specifies, that all nodes in the network must emit TC messages, including links to all their neighbor nodes. Our simulations show, that the additional link-state information available provides better robustness, against moderate node mobility: the delivery rate of data traffic starts dropping only at a higher node mobility rate than regular OLSR. The full link-state option yields, at its best, a 20\% higher delivery rate than regular OLSR, however at the expense of a $30 \%$ increase in bandwidth consumption by control traffic.

Our simulations also show, that at high mobility, the delivery rates of the MPR full link-state and the full-link state options become almost identical to that of regular OLSR.

\section{REFERENCES}

[1] L. V. Amir Qayyum and A. Laouiti. Multipoint relaying: An efficient technique for flooding in mobile wireless networks. Technical report, Project HiPERCOM, INRIA Rocquencourt, 2000. INRIA research report RR-3898.

[2] J. G. Jetcheva, D. Johnson, D. Maltz, and Y. Hu. Dynamic source routing (dsr). Internet Draft, draft-ietf-manet-dsr-06.txt, November 2001, Work in progress.

[3] G. P. Mario Gerla, Xiaoyan Hong. Fisheye state routing protocol (fsr) for ad hoc networks. Internet Draft, draft-ietf-manet-fsr-02.txt, March 2002, Work in progress.

[4] R. G. Ogier, F. L. Templin, B. Bellur, and M. G. Lewis. Topology broadcast based on reverse-path forwarding (tbrpf). Internet Draft, draft-ietf-manet-tbrpf-05.txt, March 2002, Work in progress.

[5] C. E. Perkins, E. M. Royer, and S. R. Das. Ad hoc on-demand distance vector (aodv) routing. Internet Draft, draft-ietf-manet-aodv-09.txt, November 2001, Work in progress.

[6] Philippe Jacquet, Paul Muhlethaler, Amir Qayyum, Anis Laouiti, Laurent Viennot and Thomas Clausen. Optimized Link-State Routing Protocol. Technical report, Project HiPERCOM, INRIA Rocquencourt, March 2002. draft-ieft-olsr-06.txt - work in progress.

[7] T. Clausen, P. Jacquet, A. Laouiti, P. Muhlethaler, A. Qayyum and L. Viennot. Optimized link state routing protocol. In Proceedings of IEEE INMIC, Lahore, Pakistan (INMIC 2001). MindPass Center for Distributed Systems, Aalborg University, 5th IEEE National Multi-Topic Conference (INMIC 2001), December 2001.

[8] Thomas Clausen, Gitte Hansen, Lars Christensen and
Gerd Behrmann. The optimized link state routing protocol - evaluation through experiments and simulation. In Proceeding of Wireless Personal Multimedia Communications. MindPass Center for Distributed Systems, Aalborg University, Fourth International Symposium on Wireless Personal Multimedia Communications, September 2001. 